\title{
DESIGN OF ENERGY EFFICIENT ROUTING ALGORITHM FOR WIRELESS SENSOR NETWORK (WSN) USING PASCAL GRAPH
}

\author{
Deepali Panwar ${ }^{1}$ and Subhrendu Guha Neogi ${ }^{2}$ \\ ${ }^{1}$ Department of Computer Science and Engineering, Sir Padampat Singhania \\ University, Udaipur, Rajasthan, India \\ ${ }^{1}$ deepali.panwarespsu.ac.in \\ ${ }^{2}$ subhrendu.neogiespsu.ac.in
}

\begin{abstract}
Development of energy efficient Wireless Sensor Network (WSN) routing protocol is nowadays main area of interest amongst researchers. This research is an effort in designing energy efficient Wireless Sensor Network (WSN) routing protocol under certain parameters consideration. Research report discusses various existing WSN routing protocols and propose a new WSN energy efficient routing protocol. Results show a significant improvement in life cycle of the nodes and enhancement in energy efficiency of WSN. In this paper, an attempt has been made to design a wireless sensor network involving the extraction of Pascal Graph features. The standard task involves designing a suitable topology using Pascal Graph. As per the definition of interconnection network it is equivalent that a suitable graph can represent the different computer network topologies very efficiently. Different characteristics of Pascal Graph Topology has been discovered and used in network topology design. Since Pascal Graph gives better result in terms of finding the dependable and reliable nodes in topology, it has been considered for network analysis. Moreover, we propose a methodology that involves the Pascal Graph Topology for wireless sensor network which can analyse and represent the network and help in routing.
\end{abstract}

\section{KEYWORDS}

WSN, Energy efficient, WSN routing protocol, Pascal Matrix and Pascal Graph

\section{INTRODUCTION}

Wireless Sensor Networks (WSN) is an emerging technology that could revolutionize the way wireless network access is provided. The interconnection of different wireless devices using wireless links exhibits great potential in addressing different connectivity issue. Wireless Sensor Network (WSN) is a wireless network consisting of small nodes with sensing, computation, and wireless communications capabilities [1]. Each sensor collects data from the monitored area (such as temperature, sound, vibration, pressure, motion or pollutants) and then it routes data back to the base station [2-3]. Data transmission is usually a multi- hop, from node to node toward the base station. To realize this vision, it is imperative to provide efficient resource management. Resource management encompasses a number of different issues, including routing. As wireless sensor networks consist of hundreds to thousands of low-power multi functioning sensor nodes, operating in an unattended environment, with limited computational and sensing capabilities, sensor nodes are equipped with small, often irreplaceable batteries with limited power capacity.

Rupak Bhattacharyya et al. (Eds) : ACER 2013,

pp. 175-189, 2013. (C) CS \& IT-CSCP 2013

DOI : $10.5121 /$ csit.2013.3217 
WSN consist of hundreds or thousands of small, cheap, battery-driven, spread-out nodes bearing a wireless modem to accomplish a monitoring or control task jointly. Although a profusion of routing mechanisms has been proposed for other wireless and mobile networks, the unique characteristics of WSN suggest that WSN demand a specific solution. To have a clear and precise focus on future research in WSN routing, the characteristics of WSN that have a strong impact on routing must be identified. Then a set of criteria is defined against which the existing routing protocols from ad hoc, sensor, and WSN can be evaluated and performance metrics identified. This will serve as the basis for deriving the key design features for routing in wireless sensor networks. An important concern is the network lifetime: as nodes run out of power, the connectivity decreases and the network can finally be partitioned and become dysfunctional [2-3]. Routing in WSNs is a very challenging problem due to the inherent characteristics which differentiate such networks from other wireless networks such as ad hoc networks and cellular networks [2-4]. In recent years, many algorithms have been proposed for the routing issue in WSNs. The minimum energy routing problem has been addressed [5-6]. The minimum total energy routing approaches in these papers are to minimize the total consumed energy. However, if all traffic is routed through the minimum energy path to the destination, the nodes along that path will run out of batteries quickly rending other nodes useless due to the network partition even if they do have available energy. Instead of trying to minimize the total consumed energy on the path, the objective is to maintain the connected network as long as possible. If sensor nodes consume energy more equitably, they continue to provide connectivity for longer, and the network lifetime increases [5-10]. A number of potential solutions have been proposed, including full end-to-end wireless access and ad-hoc networks. Industry has divergent views on some parameters based upon their experience of designing wireless sensor network which includes network components, properties of sensor nodes, degree of mobility, traffic pattern, energy efficiency of sensor nodes, routing between sensor nodes. The Wireless Sensor Network has been designed using different data structures such as graph, tree, linked list and other techniques like neuro-fuzzy, swarm intelligence etc. A sufficient representation has been so far made to design different computer network topologies using appropriate graph models. Graphs have huge contributions towards the performance improvement factor of a network. Some major contributors are de-Bruijn, Hypercube, Mesh and Pascal. As per the definition of interconnection network it is equivalent that a suitable graph can represent the physical and logical layout very efficiently. However, deploying these networks requires the installation of a large amount of wireless sensor devices. The initial investment costs for deployment and the difficulty of deployment in some environment settings have prevented the widespread realization of such access networks. As sensor networks have specific requirements on energy saving, data-oriented communication, and inter-connection between non-IP and IP, therefore sensor network dedicated routing protocols may be required, for energy efficient routing scheme. In WSN there are the routing protocols that minimize the used energy, extending subsequently the life span of the WSN [8-9, 11-12]. Energy awareness is an essential in routing protocol design issue. Depending on the network structure, routing in WSNs can be divided into:

a) Flat-based routing

b) Hierarchical-based routing

c) Location-based routing

Depending on the protocol operation, routing in WSNs can be divided into:

a) Multipath-based routing

b) Query-based routing

c) Negotiation-based routing

d) QoS-based routing

e) Coherent based routing 
Against this backdrop, the present study first attempts to make a comparative analysis of different graph topologies on the identified features of wireless sensor network and propose an energy efficient routing algorithm in wireless sensor network designed using Pascal graph topology. In this paper, we revisit this main idea is to design a suitable algorithm to create topology in wireless sensor network using Pascal Graph. The article is organized as follows. Section 2 briefly reviews different techniques and Section 3 deals with a precise description of our methodology. Section 4 is devoted to design issues and section 5 emphasizes on discussion of the study. Finally, Section 6 concludes the paper and gives suggestions for future work.

\section{LITERATURE REVIEW}

Architectural design issues and their implications pertaining to the Wireless Sensor Network have been observed by host of scholars. The issues related to network dynamics depends on three main components in a sensor network. These are the sensor nodes, sink and monitored events. Aside from the very few setups that utilize mobile sensors [13], most of the network architectures assume that sensor nodes are stationary .On the other hand, supporting the mobility of sinks or cluster-heads (gateways) is sometimes deemed necessary [14]. Routing messages from or to moving nodes is more challenging since route stability becomes an important optimization factor, in addition to energy, bandwidth etc. The sensed event can be either dynamic or static depending on the application [15]. Another consideration is the topological deployment of nodes. This is application dependent and affects the performance of the routing protocol. The deployment is either deterministic or self-organizing. In deterministic situations, the sensors are manually placed and data is routed through pre-determined paths. However in self-organizing systems, the sensor nodes are scattered randomly creating an infrastructure in an ad hoc manner [16-19]. During the creation of an infrastructure, the process of setting up the routes is greatly influenced by energy considerations. Since the transmission power of a wireless radio is proportional to distance squared or even higher order in the presence of obstacles, multi-hop routing will consume less energy than direct communication. However, multi-hop routing introduces significant overhead for topology management and medium access control. Direct routing would perform well enough if all the nodes were very close to the sink [17]. Most of the time sensors are scattered randomly over an area of interest and multi-hop routing becomes unavoidable. Depending on the application of the sensor network, the data delivery model to the sink can be continuous, eventdriven, query-driven and hybrid [15]. In the continuous delivery model, each sensor sends data periodically. In event-driven and query-driven models, the transmission of data is triggered when an event occurs or a query is generated by the sink. Some networks apply a hybrid model using a combination of continuous, event-driven and query-driven data delivery. The routing protocol is highly influenced by the data delivery model, especially with regard to the minimization of energy consumption and route stability. For instance, it has been concluded in [20] that for a habitat monitoring application where data is continuously transmitted to the sink, a hierarchical routing protocol is the most efficient alternative. This is due to the fact that such an application generates significant redundant data that can be aggregated on route to the sink, thus reducing traffic and saving energy. In a sensor network, different functionalities can be associated with the sensor nodes. In earlier works shown in [21-23], all sensor nodes are assumed to be homogenous, having equal capacity in terms of computation, communication and power. However, depending on the application a node can be dedicated to a particular special function such as relaying, sensing and aggregation since engaging the three functionalities at the same time on a node might quickly drain the energy of that node. Some of the hierarchical protocols proposed in the literature designate a cluster-head different from the normal sensors. While some networks have picked cluster-heads from the deployed sensors [17-19] in other applications a cluster-head is more powerful than the sensor nodes in terms of energy, bandwidth and memory [13, 18]. In such cases, the burden of transmission to the sink and aggregation is handled by the cluster-head. Since sensor nodes might generate significant redundant data, similar packets from multiple nodes can 
be aggregated so that the number of transmissions would be reduced. Data aggregation is the combination of data from different sources by using functions such as suppression (eliminating duplicates), min, max and average [24]. Some of these functions can be performed either partially or fully in each sensor node, by allowing sensor nodes to conduct in-network data reduction [22, 25-26]. Recognizing that computation would be less energy consuming than communication [17], substantial energy savings can be obtained through data aggregation. This technique has been used to achieve energy efficiency and traffic optimization in a number of routing protocols [22, 24-28]. In some network architectures, all aggregation functions are assigned to more powerful and specialized nodes [13]. Data aggregation is also feasible through signal processing techniques. In that case, it is referred as data fusion where a node is capable of producing a more accurate signal by reducing the noise and using some techniques such as beam forming to combine the signals [17]. The growing interest in wireless sensor networks and the continual emergence of new architectural techniques inspired some previous efforts for surveying the characteristics, applications and communication protocols for such a technical area[15, 23].Taxonomy of the different architectural attributes of sensor networks is developed using different models [15]. Flooding and gossiping [29] are two classical mechanisms to relay data in sensor networks without the need for any routing algorithms and topology maintenance. Although flooding is very easy to implement, it has several drawbacks [24]. Such drawbacks include implosion caused by duplicated messages sent to same node, overlap when two nodes sensing the same region send similar packets to the same neighbour and resource blindness by consuming large amount of energy without consideration for the energy constraints [29]. Gossiping avoids the problem of implosion by just selecting a random node to send the packet rather than broadcasting. However, this cause delays in propagation of data through the nodes. A growing body of literature suggests fundamental problems of topological design in computer technology [30-34]. These problems have been resolved in form of classes of Pascal graph with extreme topological parameters that correspond to the parameters of speed of information transmission, productivity, and security with limitations in costs [35]. In the phase of topological design, the computer network is represented in graph [36-37] form whose vertices correspond to the nodes of information processing, and edges correspond to the communication lines. The ontogeny of Pascal Graph (PG) was Pascal Matrix (PM) that in turn was generated meticulously from Pascal's triangle [37]. Scientists have been putting a lot of efforts in ameliorating computer network properties [38]. Wide varieties of graph model worked as resource to their brainstorming contribution in this field [39]. Pascal Graph (PG) [37] is one of those resources and played a significant role as soon as its exploration was initiated almost two decades back. As a consequence, several similar graph models emerged in this area with laudable potentials to be used as computer network topologies [37]. For reliability or security considerations, some networks may be required to provide more than one distinct path for each node pair, there by resulting in a minimum degree of connectivity between the nodes [39]. The maximum connectivity is desirable since it corresponds to not only the maximum fault tolerance of the network but also the maximum number of internally disjoint paths between any two distinct vertices. However, connectivity number can be at most equal to the degree of the network graph [40-42]. Routing in WSNs is a very challenging problem due to the inherent characteristics which differentiate such networks from other wireless networks such as ad hoc networks and cellular networks [11-12]. In recent years, many algorithms have been proposed for the routing issue in WSNs. The minimum energy routing problem has been addressed in the literature review of several scholarly articles [11-12, 43]. The routing protocols that minimize the used energy, extending subsequently the life span of the WSN [7]. The minimum total energy routing approaches in these papers are to minimize the total consumed energy. However, if all traffic is routed through the minimum energy path to the destination, the nodes along that path will run out of batteries quickly rending other nodes useless due to the network partition even if they do have available energy. Instead of trying to minimize the total consumed energy on the path, the objective is to maintain the connected network as long as possible. If sensor nodes consume 
energy more equitably, they continue to provide connectivity for longer, and the network lifetime increases [11-13].

\section{Methodology}

The proposed methodology involved in this study entails following 2 phases:

\subsection{Phase I - Deep investigation}

For achieving reliable results and excellent efficiency of the problem solution, further in depth literature review has been done. The literature review is done in section 2 .

\subsection{Phase II - Designing \& Development}

In this phase, solution of the identified problem has been developed for onward analysis, efficiency and reliability measures during modelling and simulation. The proposed solution has been reconsidered based on the analysis of the results of simulations for further improvement of the solution. Section 4 illustrates the design principles and discussed in section 5.

\section{Designing With Pascal Graph}

\subsection{Pascal Graph and method for designing topology}

An undirected graph of $\mathrm{n}$ vertices corresponding to $\mathrm{PM}(\mathrm{n})$ as an adjacency matrix is called Pascal Graph (n), where $n$ is the order of the Pascal graph [37]. The following graph has been drawn from the Pascal Matrix. The following are the example of Pascal Graph PG (3) and PG (5) derived from Pascal Matrix PM (3) and PM (5) respectively. An ( $\mathrm{x} n$ ) symmetric binary matrix is called the Pascal Matrix PM(n) of order $\mathrm{n}$ if its main diagonal entries are all 0's and its lower (and therefore the upper also) consists of the first (n-1) rows of Pascal Triangle modulo 2. Where $\mathrm{PM}_{\mathrm{i}, \mathrm{j}}$ denotes the element of $\mathrm{i}^{\text {th }}$ row and $\mathrm{j}^{\text {th }}$ column of the Pascal Matrix [37]. The topology design depends on the properties of Pascal Graph described in the section 4.2. We carry out the usual text data transformation that consists in extracting graphs of valued associations between attributes from collections of separate documents. Let us formally review this process that transforms individual data into relational data to which we can refer as network data. The Pascal Graph Topology (PGT) can provide a reliable node and shortest path with minimum of one hop count and maximum of two hops using the first node. The design paradigm is such that the first node behaves as the most dependable node but the failure of such node also creates problems in the topology. 
The following example shows the Pascal Matrix of PM (3) and PM (5) and the corresponding Pascal Graph in figure 1.

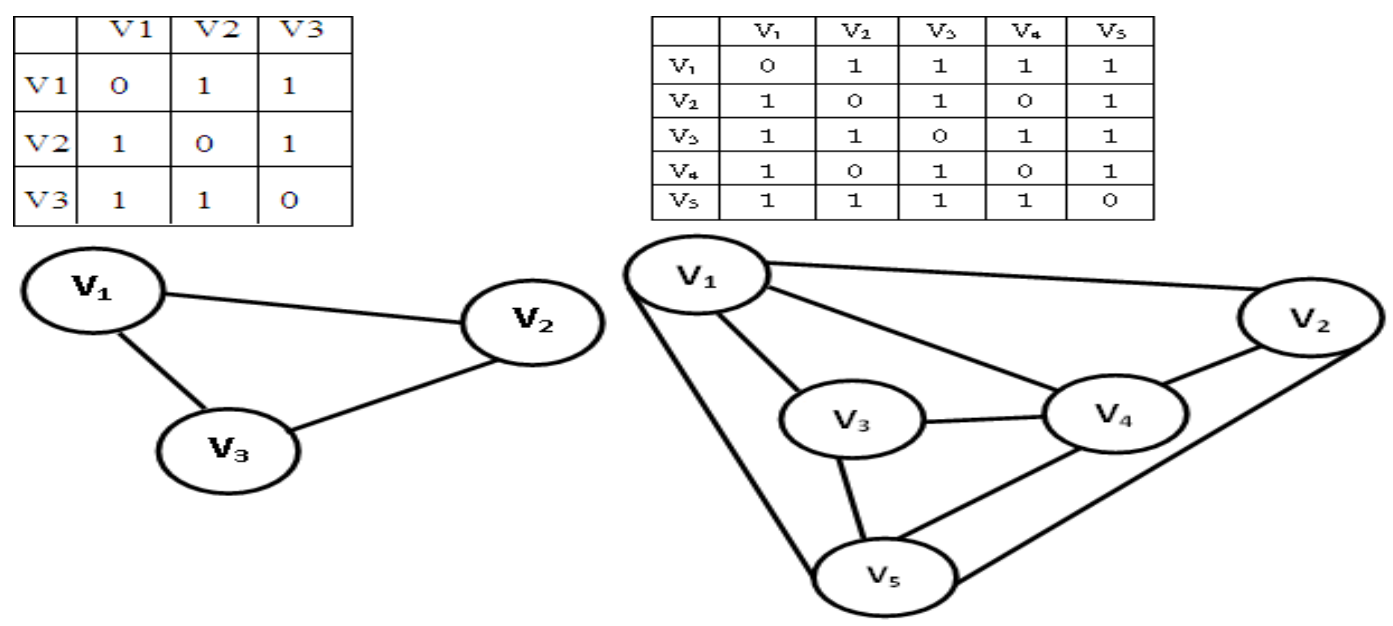

Figure 1. Pascal Graph with 3 and 5 nodes

Suppose the graph $\mathrm{G}$ has $\mathrm{n}$ vertices and $\mathrm{m}$ edges and the graph $\mathrm{G}=(\mathrm{V} ; \mathrm{E})$ denote the ad-hoc network before running the topology control algorithm, with $\mathrm{V}$ being the set of ad-hoc nodes, and $\mathrm{E}$ representing the set of communication links. The $\mathrm{n} \times \mathrm{m}$ incidence matrix of $\mathrm{G}$, denoted by IG has one row per vertex and one column per edge. The column corresponding to edge $\{i, j\}$ of IG is zero except for the $i$-th and $j$-th entries, which are $\left(E_{i j}\right)^{0.5}$ and $-\left(E_{i j}\right)^{0.5}$ respectively, where $E_{i j}$ is the corresponding edge weight. Note that there is some ambiguity in this definition, since the positions of the positive and negative entries seem arbitrary. However this ambiguity will not be important to us. The graph representation offers the advantage that it allows for a much more expressive document encoding than the more standard bag of words/phrases approach, and consequently gives improved classification accuracy. Document sets are represented as graph sets to which a weighted graph mining algorithm is applied to extract frequent sub-graphs, which are then further processed to produce feature vectors (one per document) for classification. Weighted sub-graph mining is used to ensure classification effectiveness and computational efficiency; only the most significant sub-graphs are extracted. The approach is validated and evaluated using several popular classification algorithms together with a real world textual data set. The results demonstrate that the approach can outperform existing text classification algorithms on some dataset. When the size of dataset increased, further processing on extracted frequent features is essential. In a weighted graph $\mathrm{G}=(\mathrm{V} ; \mathrm{E})$ every edge $(\mathrm{u} ; \mathrm{v})$ is attributed a weight $\omega_{\mathrm{uv}}$. When referring to a weighted graph we assume that the weights are symmetric: $\omega_{\mathrm{uv}}=\omega_{\mathrm{vu}}$. The nodes of a Euclidean graph are assumed to be located in a Euclidean plane. Furthermore the edge weight of an edge $(u ; v)$ is defined to be $\omega_{\mathrm{uv}}=$ luvl, where luvl is the Euclidean distance between the nodes $\mathrm{u}$ and v. Note that the definition of Euclidean graphs does not contain a statement on the existence of certain edges. There is a link $(\mathrm{u} ; \mathrm{v})$ in $\mathrm{E}$ if and only if the two nodes $\mathrm{u}$ and $\mathrm{v}$ can communicate directly. Running the topology control algorithm will yield a sparse sub-graph $\mathrm{G}_{\mathrm{tc}}=\left(\mathrm{V} ; \mathrm{E}_{\mathrm{tc}}\right)$ of $\mathrm{G}$, where $E_{t c}$ is the set of remaining links. The resulting topology $G_{t c}$ should be symmetric, that is, node $\mathrm{u}$ is a neighbour of node $\mathrm{v}$ if and only if node $\mathrm{v}$ is a neighbour of node $\mathrm{u}$. Although a minimum spanning tree (MST) is a sparse connected sub-graph, it is often not considered a good topology, since close-by nodes in the original graph $G$ might end up being far away in $G_{\text {tc }}(G$ being a ring, for instance). For any two nodes $u$ and $v$, if the optimal path between $u$ and $v$ in $G$ has cost $c$, then the optimal path between $u$ and $v$ in $G_{t c}$ has cost $f(c)$. If $f(c)$ is bounded from above by a linear function in $\mathrm{c}$, the graph $\mathrm{G}_{\mathrm{tc}}$ is called a spanner. Researchers have studied a selection of cost metrics, the most popular being i) Euclidean distance and ii) various energy 
metrics. The cost of a link in model i) is the Euclidean distance of the link, in model ii) the distance is raised to a predefined power. The remaining graph $\mathrm{G}_{\mathrm{tc}}$ should be sparse, that is, the number of links should be in the order of the number of nodes. It follows that from the similarity analysis point of view, the intersection graph $\mathrm{G}_{\mathrm{O}}(\mathrm{K}, \mathrm{E})$ of $\mathrm{H}$ defines the co-word graph having as many edges as there are non-null values in a similarity matrix.

\subsection{Properties of Pascal Graph and generation method}

There are certain pragmatic properties that make Pascal graph a better choice for a computer network topology over many others [40]. Some of those properties are given below:

$>\mathrm{PG}(\mathrm{n})$ is a sub-graph of $\mathrm{PG}(\mathrm{n}+1) \forall n \geq 1$ [37].

$>$ All Pascal Graph PG(i) for $i \leq 1 \leq 7$ are planner; all Pascal Graph of higher order are non-planner [44].

$>$ In the Pascal Graph, Vertex $V_{1}$ is adjacent to all other vertices and Vertex $V_{i}$ is adjacent to $\mathrm{V}_{\mathrm{i}+1}$ for $i \geq 1$ [40].

$>$ In the Pascal Graph, two even numbered vertices are never connected and adjacent to each other.

$>$ More than one node except $V_{1}$ in Pascal Graph has special properties in terms of connectivity.

$>$ If $\mathrm{k}=2 \mathrm{n}+1, \mathrm{n}$ is a positive integer and then $\mathrm{V}_{\mathrm{k}}$ is adjacent to all $\mathrm{V}_{\mathrm{i}}[44]$.

$>$ All Pascal Graph of order $\geq 3$ are 2-connected [37].

$>$ There are at least two edge disjoint path of length $\leq 2$ between any two distinct vertices in $\mathrm{PG}(\mathrm{n}), 3 \leq n$ [44].

$>$ If $V_{i}$ is adjacent to $V_{j}$, where $j$ is even and $|i-j|>1$, then i is odd and $V_{i}$ is adjacent to $V_{j-1}$ [44].

$>$ Let $\operatorname{det}(\mathrm{PM}(\mathrm{n}))$ refer to the determinant of the Pascal matrix of order $n$. Then, $\operatorname{Det}(\operatorname{PM}(n))$ is even, for all $n \geq 3$ and $\operatorname{det}(\operatorname{PM}(n))=0$, for all even $n \geq 4$ [44].

$>$ In the Pascal Graph, $V_{1}$ is considered to be most dependable and reliable node, the properties of $\mathrm{V}_{\mathrm{k}}$ have few similar properties of $\mathrm{V}_{1}$ for $\mathrm{k}=2 \mathrm{n}+1$ where $\mathrm{n}$ is a positive integer.

The following steps will generate a Pascal Graph:

Step 1: Enter p number of vertices to form a graph $G=(V ; E)$.

Step 2: Initialized LT[p,0] = 1;

Step 3: From the lower left triangle [LT $(\mathrm{p}, \mathrm{p})]$ by adding the number directly above and to the left with the number directly above and to the right to find the new value. If either the number to the right or left is not present, substitute a zero to its place [44].

Step 4: From the upper right triangle [UT(p,p)] using the same manner the generic Pascal Matrix is formed.

Step 5: Convert the lower left and upper right triangle into binary values by using modulo approach.

Step 6: From the final adjacency matrix $[\operatorname{PM}(p, p)]$ of with $L T(p, p)$ and UT(p,p).

Step 7: Stop.

If we consider a Pascal graph containing $n$ nodes i.e. $\mathrm{PG}(\mathrm{n})$ then by default $\mathrm{V}_{\mathrm{i}}(i \geq 1)$ is adjacent to all other nodes [37]. The exploration does not conclude with it as the nodes have different properties which need to be explored further. We have found and recommended other node similar to $\mathrm{V}_{1}$ in the PG(n) and termed it as Dependable Node of Pascal Graph (DNP); the special node, other than node $V_{1}$, of Pascal graph PG(n) with same degree like $V_{1}$ [44]. The node $V_{1}$ is 
called most dependable and reliable node in terms of routing and path optimization in the graph. To satiate our claim and to rationalize the whole thing we have used very simplistic approach [40, $41]$.

The explanation with some suitable example is given below.

Case 1: $m=2(\log m)$

Case 2: $m \pi 2[\log m]$

Case N: $m=2[\log m]+1$

To establish the new property, we are supposed to generate the index i of DNP.

For Case 1: $i=2(\log m)-1+1$, where $i \phi 1$

For Case 2: $i=2[\log m]+1$ where $i \phi 1$

For Case $\mathrm{N}: i=2[\log m]-1+1$ and $i=2[\log m]+1$, where $i \phi 1$

The formation of Pascal graph depends on DNP and its properties. The graph has mesh network but minimum number of connectivity as per requirements. There are atleast two paths available between nodes and $\mathrm{V}_{1}$ can provide connectivity with minimum hop count between any nodes. The similar property can be found with the nodes have same functionality with $V_{1}$ as per the formation of graph.

The design of Pascal Graph from Pascal Matrix showed the existence of Dependable nodes of Pascal Graph (DNP) along with $\mathrm{V}_{1}$ where there is a full connectivity with other nodes. The graphs are not planner in the case of $\mathrm{PG}(\mathrm{n})$ with $\mathrm{N}>7$ but the connectivity is not as complex as we have more than two dependable nodes in some cases. The Table 1 gives the list of DNP in case of Pascal Graph where $\mathrm{n}>7$.

Table 1. List of DNP

\begin{tabular}{|l|l|l|l|}
\hline PG(n) & i & DNP & Degree \\
\hline 8 & 5 & V5 & 7 \\
\hline 9 & 5,9 & V5, V9 & 8 \\
\hline 10 & 9 & V9 & 9 \\
\hline 11 & 9 & V9 & 10 \\
\hline 12 & 9 & V9 & 11 \\
\hline 13 & 9 & V9 & 12 \\
\hline 14 & 9 & V9 & 13 \\
\hline 15 & 9 & V9 & 14 \\
\hline 16 & 9 & V9 & 15 \\
\hline
\end{tabular}

\section{DISCUSSION}

There are many other studies related to design using graph. Geibel and Wysotzki proposed a method to derive induced sub-graphs of graph data and to use the induced sub-graphs as attributes on decision tree approaches [45-46]. Their method can be used to find frequent induced subgraphs in the set of graph data. However, the upper limit number of the vertices to be included in the sub-graph must be initially specified to avoid the exponential explosion of the computational time, and thus the search is not complete. Liquiere and Sallantin proposed a method to completely search homomorphically equivalent sub-graphs which are the least general over a given set of 
graphs and do not include any identical triplet of the labels of two vertices and the edge direction between the vertices within each sub-graph [47].

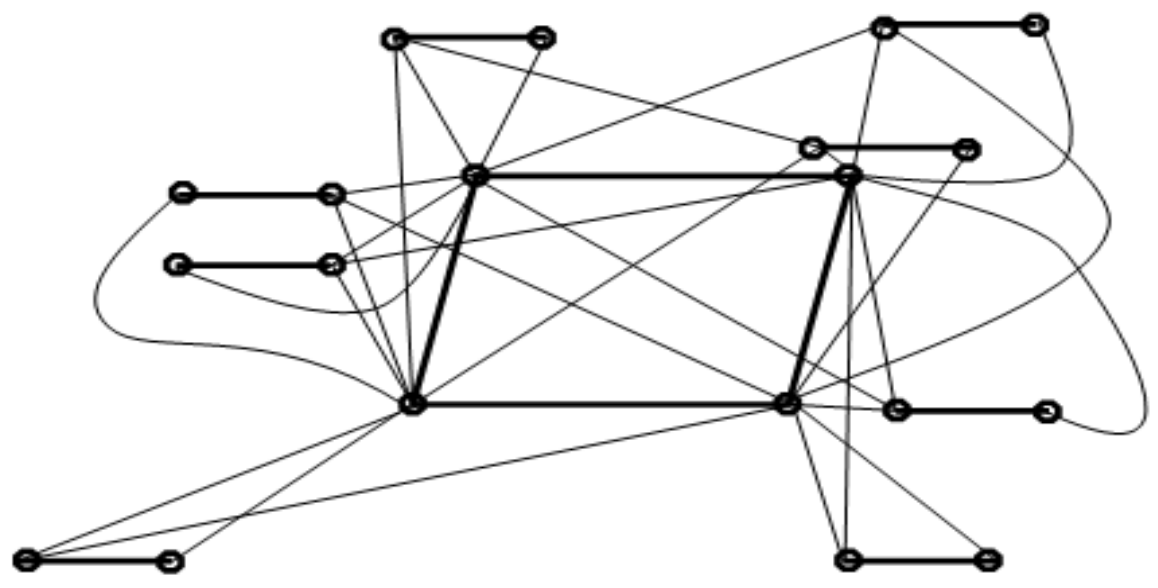

Figure 2. Mesh-Pascal(a,b,n)-Graph

From the Figure 2, we can find that it is forming a well-connected graph compared to other graphs. Pascal graph is best among them in terms of diameter but it is worse in terms of maximum degree of a node and total number of edges. On the other hand, ring and mesh perform well in terms of maximum degree of a node and total number of edges, but they both perform poorly in terms of diameter of graph. The proposed graph gives a standard measurement in terms of degree, diameter and total number of edges. The only graph which comes close to it is hypercube graph. But the hypergraph fails measurably compared to the proposed graph model when we consider total number of edges, reliability of the network and expansion-contraction of the network. If we expand the hypercube from the dimension $q$ to $q+1$, it would reconfigure $2 q$ number of nodes (which increases exponentially). But the proposed model improved by adding nodes to PGN (Pascal Graph Network), so we have to reconfigure a few member of nodes to get a better network as in Mesh-Pascal Graph(ab,n), multiple path exists between two nodes and the network can be more reliable and fault tolerant for that reason.

The proposed model is good enough with comparison to other WSN network model since it gives minimal degree and diameter but maximum reliability and expansion. The entire simulation tested by using a very well-known simulator NS2 where different Pascal Graph topology has been designed. The three topologies shown in figures 3,4,5 below; where we have tested with 2 nodes, 3 nodes and 4 nodes for the testing of Pascal Graph property. We have also tested with more number of nodes as per the topology shown in Figure 1. The different network protocols has been designed and implemented in NS2 simulator. NS2 includes a tool for viewing the simulation results, called NAM; NAM is an animation tool for viewing network simulation traces and real world packet data. The first step to use NAM is to produce trace file containing topology information for nodes and links which generated by NS Tcl/Tk script. During the simulation, we have designed topology configuration and layout information for Pascal Graph, tracing events using NS and visualize with NAM. Based on the simulation various graphs has been plotted to demonstrate the performance of Pascal Graph. We have tried to show the results of the simulated routing and sensor node energy. Using the same topology we have compared Pascal graph network with existing WSN network model using NS2. The graph shows the performance of PGN and the routing is better in comparison with other WSN network since the first node is effective for minimum cost routing in terms of different factors of energy efficient node optimized routing mechanism. PGN always tries to find shortest path using first node which is also called most dependable node. This is one of the major drawbacks of PGN. So the energy 
consumption by the nodes which has higher connectivity is more and they are dependable nodes as well. So we considered the energy efficiency of dependable nodes and the performance also being demonstrated in time frame and it is one of the major issues in PGN that the energy consumption is higher in case of DNP and this can be one of the major factors of routing as well. The following figures illustrate the PGN and performance of the network designed in NS2 simulator.

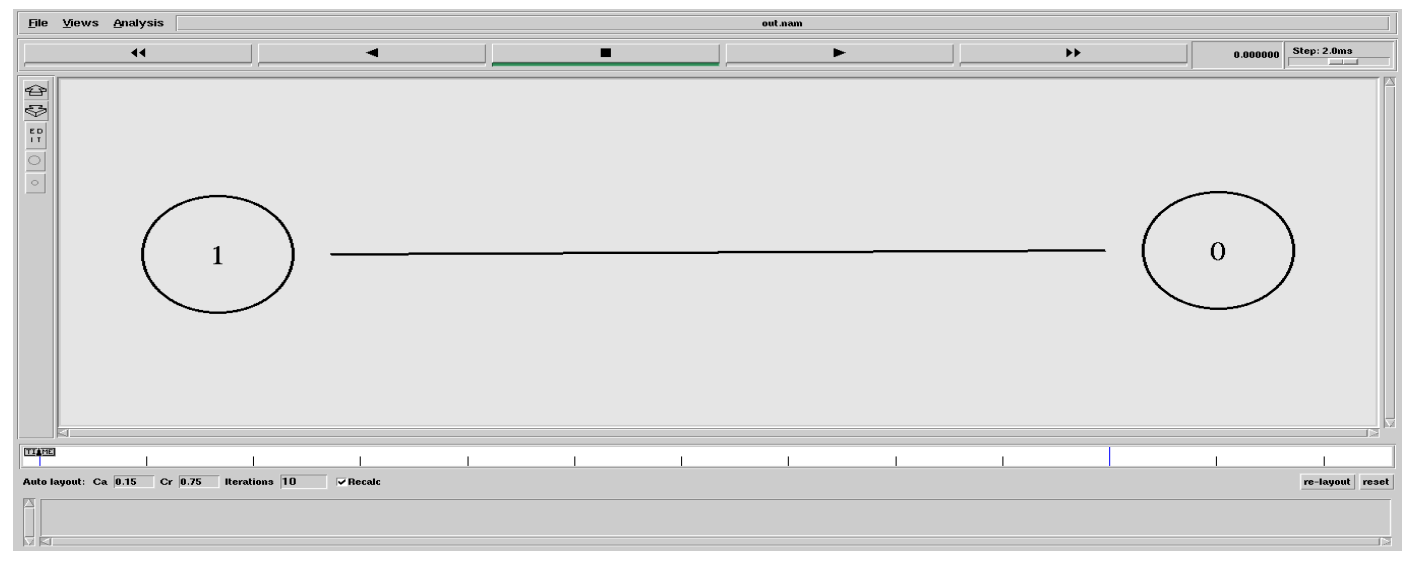

Figure 3. Pascal Graph with two nodes

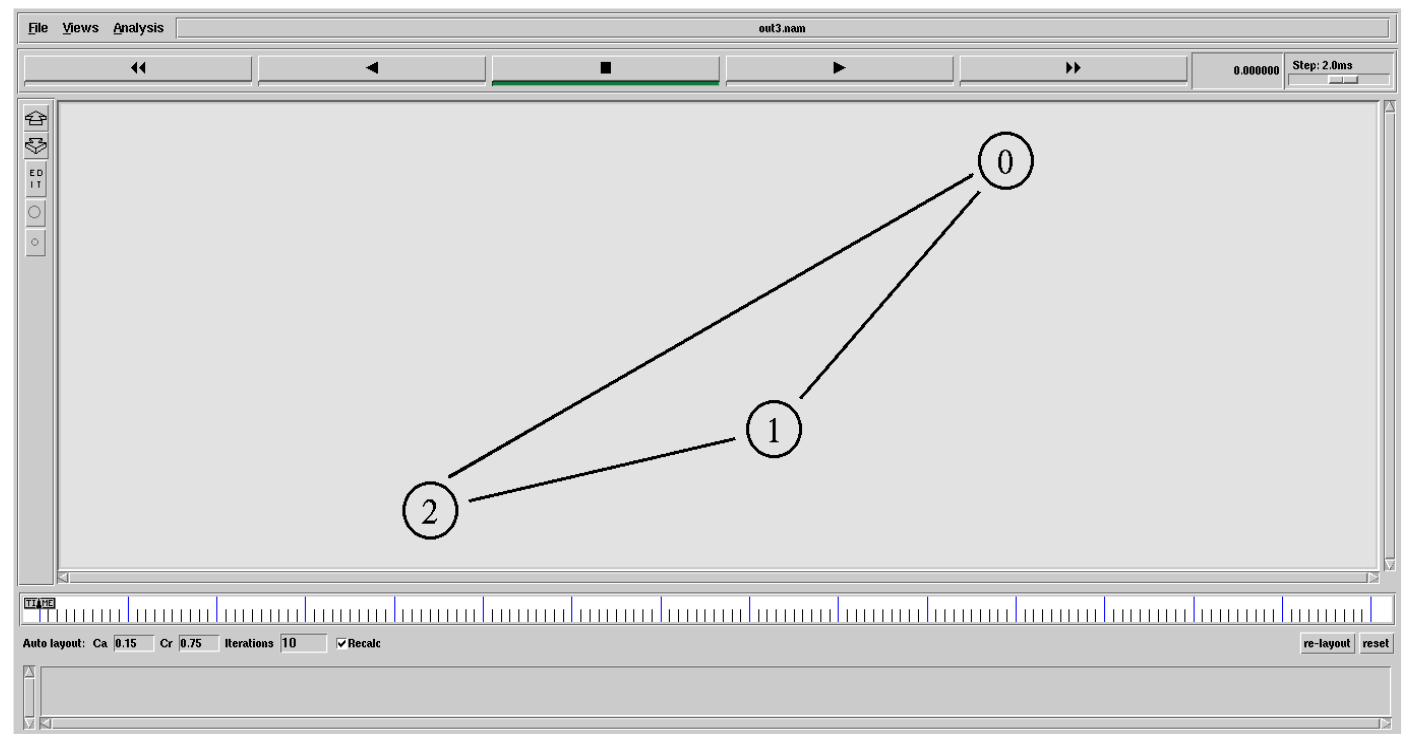

Figure 4. Pascal Graph with three nodes 


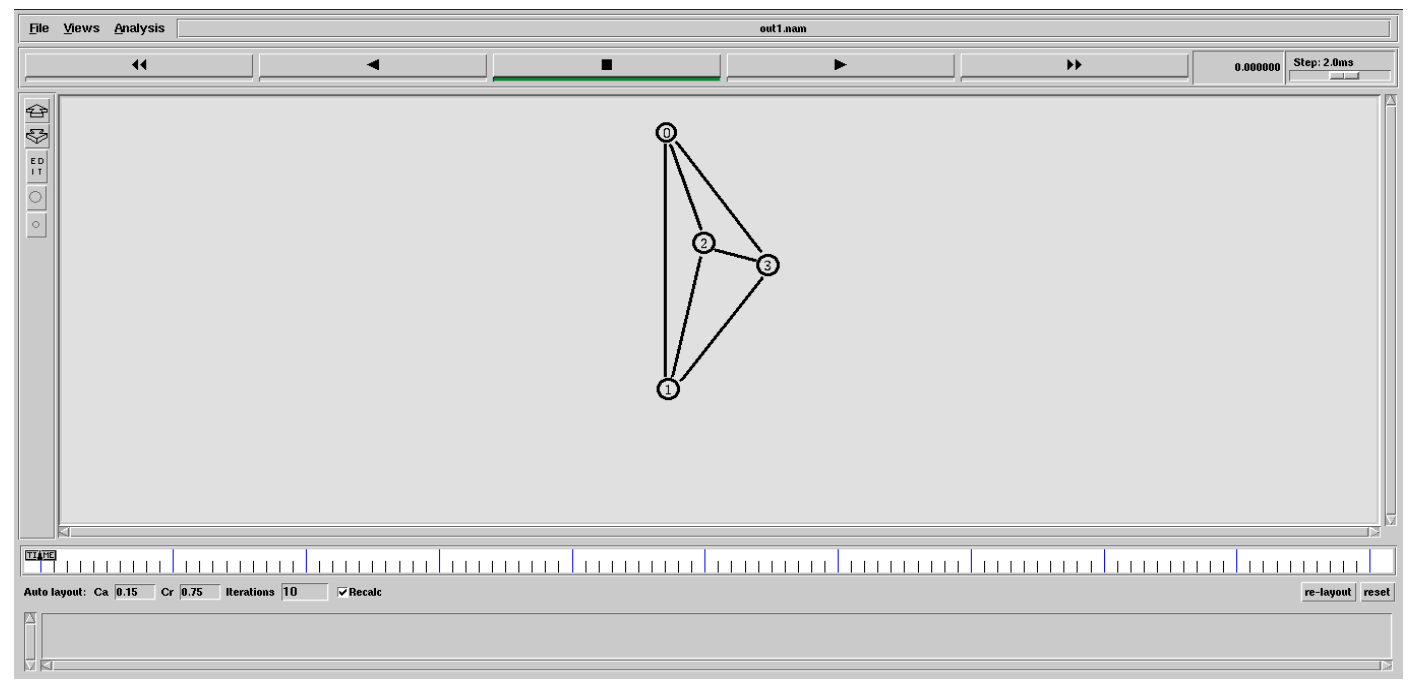

Figure 5. Pascal Graph with four nodes

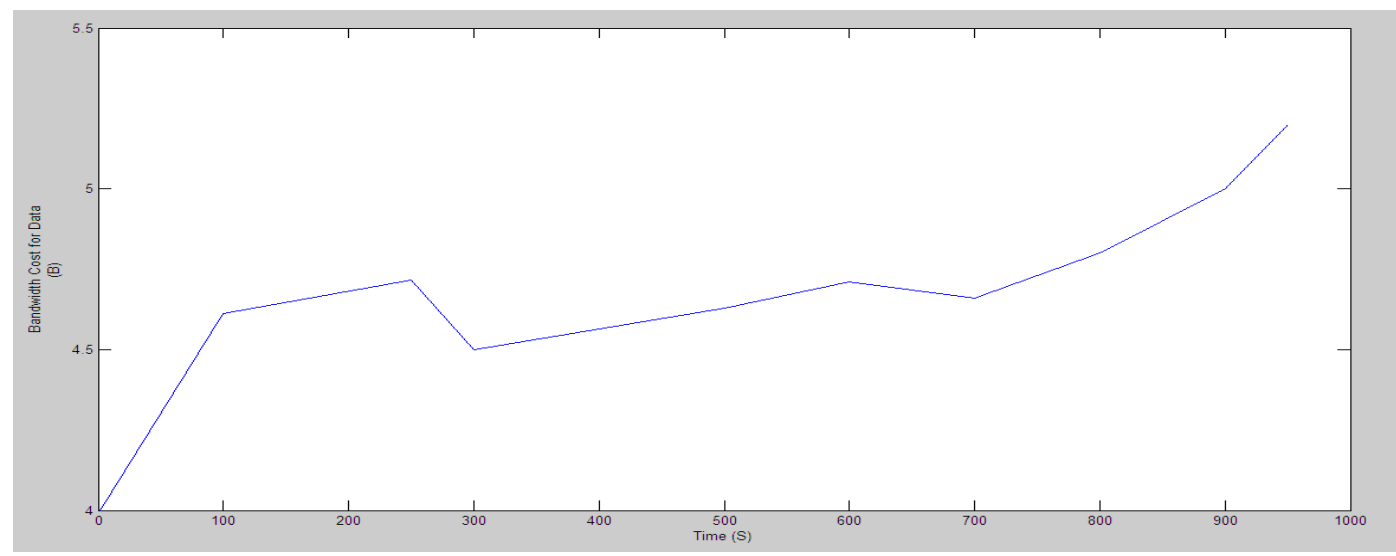

Figure 6. The bandwidth cost for data

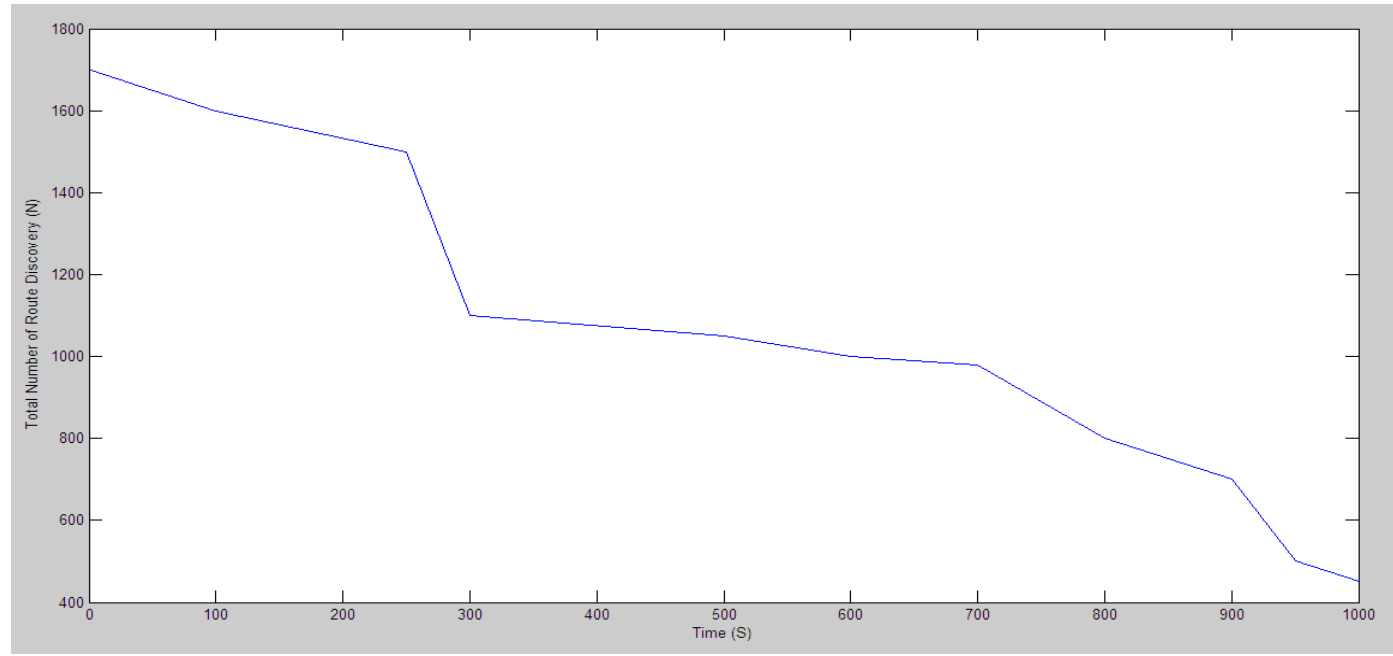

Figure 7. The number of route discovery 


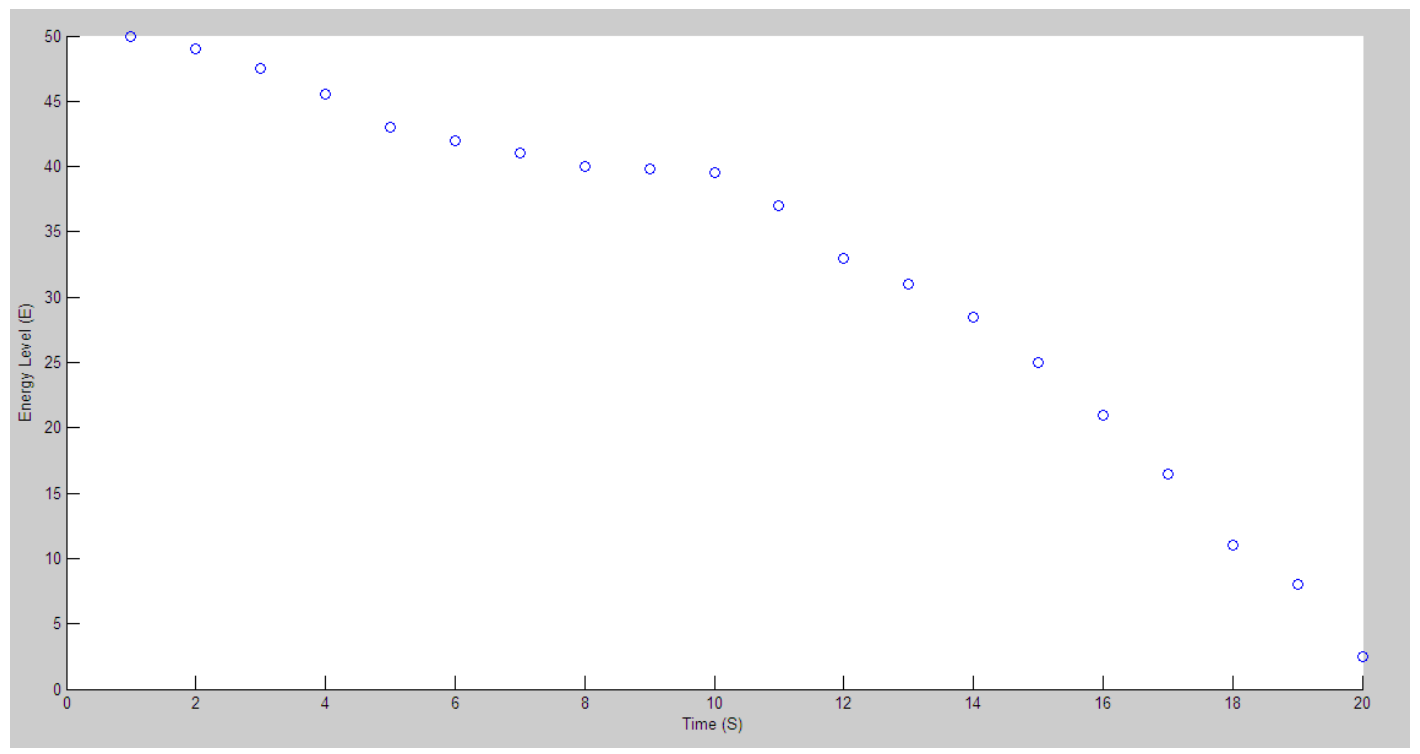

Figure 8. WSN node life and energy drain with respect to time after increasing hops

\section{ConClusion AND Future Scope OF Study}

In this paper we have proposed new WSN routing algorithm by using Pascal Graph. Many new routing and MAC layer protocols and different techniques have been proposed for WSN network and most of them trying to resolve the resource constrained for unattended wireless sensor environment, The majority of all the protocols mainly Concentrate on energy efficiency of sensor nodes, however sensor application have very important role specially in critical applications like the defence and health where the accuracy and guaranteed data transfer timely is an important issue. In the same way with some more specific sensor applications where the data type is mainly image and movie is supposed to be transfer, we required more accuracy and guarantee for timely data transfer. Hence transfer of data in such cases mainly requires QoS aware routing network management in order to ensure efficient usage of the sensor nodes. We already start working on a proposed and approved project from Deanship of scientific research, KFU, related to the QoS of WSN as it has a very key role for enhancing WSN efficiency and even its life, with that project we will focus on operational and architectural challenges of handling QoS routing traffic in sensor network and will propose a new mechanism for QoS based routing protocol to further enhance WSN life. On other hand, as we know that wireless sensor network (WSN) becomes more attractive area for the researchers now day, due to the wide range of its application areas and due to the distributed and deployment nature of these networks in remote areas, these networks are vulnerable to numerous security threats that can adversely affect their proper functioning. This problem is more critical if the network is deployed for some mission-critical applications such as in a tactical battlefield. Random failure of nodes is also very likely in reallife deployment scenarios.

\section{ACKNOWLEDGEMENTS}

Authors would like to thank their colleagues and students of Sir Padampat Singhania University for their valuable help and support. 


\section{REFERENCES}

[1] Jamal N. Al-Karaki, Ahmed E Kamal, (2004), "Routing Techniques in Wireless Sensor Network: A Survey", IEEE Wireless Communication, accessed at http://www.ics.uci.edu/ dsm/ics280sensor/readings/networks/routing-survey.pdf on 1.1.2013.

[2] I.F. Akyildiz, W. Su, Y. Sankarasubramaniam, and E. Cayirci, (2002), "A Survey on Sensor Network", IEEE Communication Magazine, vol. 40, no.8, pp. 102 - 116.

[3] N. Bulusu, J. Heidemann, and D. Estrin, (2000), "GPS-less Low Cost Out Door Localization for Very Small Devices,” Tech. rep. 00729, Comp. Sci. Dept., USC, Vol. 7(5), pp. 28 - 34.

[4] Q. Cao, T. Abdelzaher, T. He, and R. Kravets, (2007), "Cluster-Based Forwarding for Reliable Endto-End Delivery in Wireless Sensor Networks", 6th IEEE International Conference on Computer Communications. IEEE, vol., no., pp. 1928-1936.

[5] Ettus, M., (1998), "System Capacity, Latency, and power consumption in multihop-routed SSCDMA wireless networks", Proc. of RAWCON'98, pp. 55-58.

[6] Rodoplu, V. and T. H. Meng, (1999), "Minimum energy Mobile wireless networks", IEEE JSAC, vol.17, no.8, pp. 1333-1344.

[7] Bhardwaj, M. and A. Chandrakasan, (2002), "Bounding the lifetime of sensor networks via optimal role, assignments", Proc. of the INFOCOM'02, pp. 1587-1596.

[8] Chang. J H., Tassiulas, L., (2000), "Energy conserving routing in wireless ad-hoc networks", INFOCOM, Nineteenth Annual Joint Conference of the IEEE Computer and Communications Societies Proceedings, IEEE, vol.1, no., pp.22-31.

[9] Chang, J. H. and L. Tassiulas, (2004), "Maximum lifetime routing in wireless sensor networks", IEEE/ACM Trans. on Networking, vol.12, no.4, pp. 609-619.

[10] Zussman, G. and A. Segall, (2003), "Energy efficient routing in Ad Hoc disaster recovery networks", Proc. of the INFOCOM'03, pp.682-691.

[11] A. K. Sadek, W. Su, and K. J. R. Liu, (2007), "Multi node cooperative communications in wireless networks," IEEE Trans. Signal Processing, vol. 55, no. 1, pp. 341-355, 2007.

[12] A. K. Sadek, Z. Han, and K. J. Ray Liu, (2006), "A distributed relay-assignment algorithm for cooperative communications in wireless networks," in Proc. IEEE International Conference on Communications (ICC), vol. 4, pp. 1592-1597.

[13] Subramanian, L., Katz, R.H., (2000), "An architecture for building self-configurable systems", in: Proceedings of IEEE/ACM Workshop on Mobile Ad Hoc Networking and Computing, pp. 63-72.

[14] Ye,F., et al., (2002), "A two-tier data dissemination model for large scale wireless sensor networks", in: Proceedings of the 8th annual international conference on Mobile computing and networking, pp. 148-159.

[15] Tilak, S., et al., (2002), "A taxonomy of wireless micro sensor network models, Mobile Computing and Communications", Review 6 (2), pp. 28-36.

[16] Sohrabi, K., et al., (2000), "Protocols for self-organization of awireless sensor network", IEEE Personal Communications 7(5), pp. 16-27.

[17] Heinzelman, W, Chandrakasan, A., Balakrishnan, H., (2000), "Energy-efficient communication protocol for wireless sensor networks", in: Proceeding of the Hawaii International Conference System Sciences, Hawaii, 8(8), pp. 929-942.

[18] Younis, M., Youssef, M., Arisha, K., (2002), "Energy-aware routing in cluster-based sensor networks," Modeling, Analysis and Simulation of Computer and Telecommunications Systems, 2002. MASCOTS 2002. Proceedings. 10th IEEE International Symposium on , vol., no., pp. 129- 136.

[19] Manjeshwar A, Grawal DP. TEEN: A protocol for enhanced efficiency in wireless sensor networks. Proceeding of the 15th Parallel and Distributed Processing Symp. San Francisco: IEEE Computer Society, 2001, pp. 2009-2015.

[20] Heinzelman, W., (2000), "Application specific protocol architectures for wireless networks", PhD Thesis, MIT, accessed at http://www.sigmobile.org/phd/2000/theses/heinzelman.pdf on 1.1.2013.

[21] Katz, R. H., Kahn, J. M., Pister, K. S. J., (1999), "Next century challenges: mobile networking for "Smart Dust" ", in: Proceedings of the 5th annual ACM/IEEE international conference on Mobile computing and networking, pp. 271-278.

[22] Intanagonwiwat, C., Govindan, R., Estrin, D., (2000), "Directed diffusion: a scalable and robust communication paradigm for sensor networks", in: Proceedings of the 6th annual international conference on Mobile computing and Networking, pp. 56-67. 
[23] Estrin, D., et al., (1999), "Next century challenges: scalable coordination in sensor networks", in: Proceedings of the 5th annual ACM/IEEE international conference on Mobile computing and networking, pp. 263-270.

[24] Heinzelman, W., Kulik, J., Balakrishnan, H., (1999), "Adaptive protocols for information dissemination in wireless sensor networks", in: Proceedings of the 5th annual ACM/IEEE international conference on Mobile computing and networking, pp. 174-185.

[25] Lindsey, S., Raghavendra, C. S., (2002), "PEGASIS: Power-efficient gathering in sensor information systems," Aerospace Conference Proceedings, IEEE, vol.3, no., pp. 3-1125- 3-1130.

[26] Krishnamachari, B., Estrin, D., Wicker, S., (2002), "Modeling data centric routing in wireless sensor networks", in: Proceedings of IEEE INFOCOM, New York, pp 142.

[27] Braginsky, D., Estrin, D., (2002), "Rumor routing algorithm for sensor networks", in: Proceedings of the First Workshop on Sensor Networks and Applications (WSNA), Atlanta, GA., accessed at www.cs.wmich.edu/wsn/doc/rumor/Rumor.pdf on 1.1.2013.

[28] Schurgers, C., Srivastava, M. B., (2001), "Energy efficient routing in wireless sensor networks," Military Communications Conference, 2001. MILCOM, Communications for Network-Centric Operations: Creating the Information Force, IEEE, vol.1, no., pp. 357- 36.

[29] Akyildiz,I.F., et al., (2002), "Wireless sensor networks: a survey", Computer Networks 38 (4), $393-$ 422.

[30] Hedetniemi,S., Liestman,A., (1998), "A survey of gossiping and broadcasting in communication networks", Networks 18 (4), 319-349.

[31] Shah, R.C.; Rabaey, J.M., (2002), "Energy aware routing for low energy ad hoc sensor networks," Wireless Communications and Networking Conference, WCNC2002, IEEE, vol.1, no., pp. 350- 355.

[32] Petrek. J., (2002), "A New Assignment Algorithm for Star Network Topology Design", In: 9th IEEE International Conference on Electronics, Circuits and Systems ICECS 2002. Dubrovnik. Croatia, 15.18.9.., pp. 789-792.

[33] Latha. S. \& Srivastava., S. K., (2007), "On Some Aspects of Design of Cheapest Survivable Networks", IJCSNS International Journal of Computer Science and Network Security, VOL.7 No.11, pp. 210.

[34] Kamalesh., V. N, \& Srivastava., S. K., (2009), "Topological Design of Minimum Cost Survivable Computer Communication Networks: Bipartite Graph Method", International Journal of Computer Science and Information Security, Vol. 3, No. 1, accessed at http://arxiv.org/pdf/0908.1033 on 1.1.2013.

[35] Berna. D., et al., (1995), "A Genetic Algorithm Approach to Optimal Topological Design of All Terminal Networks, Proceedings of the Artificial Neural Networks in Engineering Conference ANNIE`95, University of Missouri-Rolla, St. Louis, Missouri, USA, Nov.12-15, pp. 405-411.

[36] Mason., L., et al., (2006), "Topological Design and Dimensioning of Agile All Photonic Networks", accessed at http://www.tsp.ece.mcgill.ca/Networks/projects/pdf/ mason_JournalCompComm06.pdf, on 1.1.2013.

[37] Deo, N., and Quinn, Michael J. (1983), "Pascal Graphs and their properties", Fibonacci Quarterly, Vol. 21, No. 3: pp $203-214$.

[38] Mir, N. F., (2002), “Analysis of High Speed Interconnection Network", Journal of Interconnection Networks, vol. 3, pp. 49-65.

[39] Bhattacharya, U. and Chaki, R., (2000), "A New Scalable Topology for Multihop Optical Network" ASIAN, pp. 263-272.

[40] Chatterjee, S., Sarma, S. S., (2004), "In Search Of A Versatile And Flexible Graph Model To Implement Computer Network", Proceedings of International Conference on Communications, Devices and Intelligent Systems (CODIS): pp. $635-638$.

[41] Kamalesh, V. N and Srivatsa,S. K., (2007), "Numbering of nodes in computer communication networks: A brief review", National Conference on Advanced Network Technologies and Security Issues, FISAT, Cochin, Kerala, India, pp. 278-282.

[42] West, D. B, (2001), "Introduction to Graph Theory", Pearson Education, inc 2nd Edition.

[43] Xu, J., (2002), "Topological Structure and Analysis of Interconnection Networks", Kluwer Academic Publishers.

[44] Pal, S. and Sarma, S. S., (2010), "Computer Network Topology Design in Limelight of Pascal Graph Property", International Journal of Next-Generation Networks 2.1, pp. 30-35.

[45] Watts, D.J. (1999), "Small Worlds: The Dynamics of Networks between Order and Randomness", Princeton, NJ: Princeton University Press, pp. 266. 
[46] Geibel, P and Wysotzki, F., (1996), "Learning relational concepts with decision trees", In ICML'96: 13th Int. Conf. Machine Learning, pp. 166-174.

[47] Liquiere, M. and Sallantin, J., (1998), "Structural machine learning with galois lattice and graphs", In ICML'98: 15th Int. Conf. Machine Learning, pp. 305-313.

\section{AUTHORS}

Ms. Deepali Panwar is currently a M.Tech student in the department of Computer Science and Engineering of Sir Padampat Singhania University. She has received B.Tech with honours degree from Rajasthan Technical University in the year 2010. She has teaching experience of one year from Govt. polytechnic college, kota. Her research interest lies in Wireless Sensor Networks.

Mr. Subhrendu Guha Neogi is currently working as Assistant Professor in the depa rtment of Computer Science and Engineering of Sir Padampat Singhania University. He has been awarded Masters in Engineering from West Bengal University of Technology. $\mathrm{He}$ has more than 10 years of experience in teaching. He served many reputed educational Institutes in India in various positions.

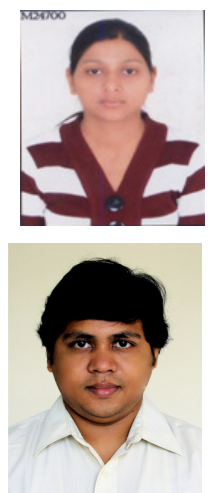

